\title{
Utilização de eletroquimioterapia em neoplasias de origem epitelial ou mesenquimal localizadas em pele ou mucosas de cães
}

\author{
Lucia Maria Guedes SILVEIRA ${ }^{1}$ \\ Carlos Henrique Maciel \\ BRUNNER ${ }^{1}$ \\ Fernando Malagutti CUNHA ${ }^{1}$ \\ Fabio FUTEMA ${ }^{1}$ \\ Franco Ferraro CALDERARO ${ }^{1}$ \\ Didio KOZLOWSKI ${ }^{2}$
}

Correspondência para:

Lucia M G Silveira, Rua Paulo Franco, 142, Apartamento 151, Vila Leopoldina. CEP: 05305-030, São Paulo, SP

Imgsilveira@hotmail.com

Recebido para publicação: 19/03/2009 Aprovado para publicação: 07/01/2010

\author{
1- Departamento de Medicina Veterinária da Universidade Paulista, São \\ Paulo-SP \\ 2- Engenheiro eletrônico, Cromática Ltda, São Paulo-SP
}

\section{Resumo}

A eletroquimioterapia é caracterizada como um protocolo que agrega o uso de fármacos antineoplásicos à aplicação regional de pulsos elétricos, maximizando a concentração intracelular destes agentes, assim propiciando maior ação citotóxica dos mesmos. A bleomicina, um antimicrobiano dotado de propriedade antineoplásica, demonstra restrição no transporte através da membrana celular, dada sua composição molecular hidrofílica. Todavia, uma vez administrada via intralesional ou endovenosa associada à eletroporação, exibe citotoxicidade potencializada. Foram utilizados neste estudo 34 cães acometidos por neoformações solitárias de origem epitelial ou mesenquimal, situadas em pele ou membranas mucosas. Padronizou-se o protocolo eletroquimioterápico empregando-se sulfato de bleomicina, pela via intralesional, na dose de $1 \mathrm{U} / \mathrm{cm}^{3} \mathrm{de}$ tumor. A eletroporação foi perfilada com eletrodo composto por agulhas, pulsos elétricos com tensão de $1000 \mathrm{~V}$, em onda quadrada unipolar, com duração de 100 microsegundos, totalizando-se oito ciclos. Constatou-se remissão neoplásica integral em 30 cães (88,3\%) e refratariedade ao protocolo em apenas quatro animais (11,7\%). Inexistiram complicações e/ou efeitos adversos decorrentes do procedimento. O protocolo neste trabalho estudado revelou-se aplicável, eficaz e seguro na terapêutica antineoplásica em cães.
Palavras-chave:

Cão.

Neoplasia.

Eletroporação.

Eletroquimioterapia.

\section{Introdução}

A oncologia constitui uma especialidade médica centrada no estudo das enfermidades neoplásicas que atualmente disponibiliza diversos protocolos, por vezes multidisciplinares, destinados à terapêutica antitumoral. Todavia, tais procedimentos exibem expressivas disparidades relativamente à eficácia, periodo de tratamento, restabelecimento orgânico do paciente, segurança e onerosidade ${ }^{1}$.

A utilizaçáo de corrente elétrica na terapêutica antineoplásica data de meados do século dezenove². O método físico embasado na administração regional de pulsos elétricos breves e de alta intensidade é designado como eletroporação. Tal princípio apresenta como propriedade intrínseca, a gênese de poros transitórios, seletivos e reversíveis na membrana celular, os quais otimizam a veiculação de substâncias químicas, ácidos nucleicos, anticorpos e plasmídeos, dentre outros elementos, ao meio intracelular $r^{3,4,5,6,7,8}$.

A eletroquimioterapia caracteriza-se pela associação de agentes antineoplásicos à eletroporação, com o intuito de se maximizar a concentração intracelular destes fármacos, assim propiciando maior ação citotóxica $9,10,11,12,13,14,15,16$. Múltiplos 
quimioterápicos, por apresentarem-se como moléculas hidrofílicas, exibem dificuldade em atravessar a membrana celular. Dentre os mencionados agentes, destaca-se a amplificaçáo da citotoxicidade exercida pela bleomicina quando combinada à aplicação regional de pulsos elétricos ${ }^{3,4,9,14,16,17}$.

Segundo a bibliografia consultada, a eletroquimioterapia representa protocolo antineoplásico com pletora de indicaçóes na clínica oncológica, exceto para tumores de origem óssea e hematopoiética ${ }^{9,12,14,17,18,19,20,21}$. De acordo com os trabalhos reunidos, tal procedimento revelou resultados satisfatórios, sobretudo caracterizados por elevado índice de remissão neoplásica, pequeno percentil de recidivas e/ou metástases adjacentes e/ou distantes e ainda efeitos adversos inexpressivos $3,9,12,14,16,17,18,19,20,21,22$.

Este trabalho objetivou avaliar a aplicabilidade, eficácia e segurança da eletroquimioterapia em neoplasias de origem epitelial ou mesenquimal, localizadas em pele ou mucosas de cáes, no intuito da caracterização das vantagens intrínsecas ao protocolo.

\section{Materiais e Métodos}

Foram utilizados 34 cães acometidos por neoformaçôes solitárias de origem epitelial ou mesenquimal, situadas em pele ou membranas mucosas, admitidos junto ao Serviço de Cirurgia do Hospital Veterinário da Faculdade de Medicina Veterinária da Universidade Paulista, São Paulo, Capital. O estudo foi conduzido respeitando-se diretrizes referentes à experimentação animal, pré-definidas pelo Conselho de Ética da Universidade Paulista (protocolo número 024/08, aprovado e registrado em ata de reuniáo do Conselho de Ética em 12 de fevereiro de 2009). Os proprietários foram previamente orientados sobre os procedimentos relativos ao protocolo.

Todos os pacientes foram avaliados por exame físico e complementares, os últimos representados por hematológico, bioquimicos, radiográfico torácico e sonográfico abdominal, no intuito da pesquisa por enfermidade sistêmica coexistente e/ ou neoplasia propagada para espaço(s) cavitário(s). Utilizou-se de técnica incisional para colheita de fragmentos das lesóes, imediatamente fixados em solução tamponada de formalina a $10 \%$ para posterior submissão à análise histológica. Todos os indivíduos da amostragem considerada receberam anestesia geral para realizaçáo de parte dos procedimentos relacionados (diagnóstico por biopsia incisional e terapêutico).

A localização e extensão dos tumores foram caracterizadas em concordância com o sistema de estadiamento clínico TNM (tumor / linfonodo / metástase), segundo diretrizes estabelecidas pela Organização Mundial da Saúde (Quadros 1 e 2) 23,24.

Padronizou-se o protocolo eletroquimioterápico utilizando-se sulfato de bleomicina (Tecnomicina ${ }^{\oplus}$ - Zodiac Produtos Farmacêuticos S. A. Pindamonhangaba, São Paulo), pela via intralesional, na dose de $1 \mathrm{U} / \mathrm{cm}^{3}$ de tumor. $\mathrm{O}$ volume neoplásico foi calculado com auxílio de paquímetro, respeitando-se premissas da equação altura $\mathrm{x}$ largura $\mathrm{x}$ profundidade $\mathrm{x} \pi / 6$. Três minutos após a aplicação do fármaco pela via supracitada, administraram-se pulsos elétricos sobre toda extensão da neoformação por meio de aparelhagem destinada a tal fim (Eletroporador Crown Modelo BK100 Cromática Ltda. São Paulo, São Paulo). A eletroporação foi perfilada com eletrodo composto por oito agulhas de aço, paralelamente dispostas e equidistantes $(0,7$ $\mathrm{cm})$. Pulsos elétricos com tensão de $1000 \mathrm{~V}$, em onda quadrada unipolar, com duraçáo de 100 microsegundos, totalizando-se oito ciclos, foram regionalmente aplicados. $\mathrm{O}$ procedimento foi repetido em intervalos mensais até que a remissão neoplásica integral fosse verificada.

Todos os animais foram reavaliados aos sete, 14,21 e 30 dias da realização do protocolo, e após regressão tumoral completa, monitorados por meio de retornos bimestrais durante 12 meses. 


\begin{tabular}{|c|c|}
\hline \multicolumn{2}{|c|}{ Tumor primário $(\mathrm{T})$} \\
\hline Tis: & Tumor pré invasivo (in situ) \\
\hline T0: & Sem evidências de tumor \\
\hline T1: & Tumor $<2 \mathrm{~cm}$ de diâmetro \\
\hline T2: & Tumor entre 2 - $5 \mathrm{~cm}$ de diâmetro \\
\hline T3: & Tumor $>5 \mathrm{~cm}$ de diâmetro \\
\hline T4: & Tumor invadindo outras estruturas como fáscia, osso ou cartilagem \\
\hline \multicolumn{2}{|c|}{ Linfonodo $(\mathrm{N})$} \\
\hline N0: & Sem evidências de envolvimento de linfonodo regional \\
\hline \multirow[t]{3}{*}{ N1: } & Linfonodo ipsilateral móvel \\
\hline & N1a: sem aumento de volume \\
\hline & N1b: com aumento de volume \\
\hline \multirow[t]{3}{*}{ N2: } & Linfonodo contralateral ou bilateral móveis \\
\hline & N2a: sem aumento de volume \\
\hline & N2b: com aumento de volume \\
\hline N3: & Linfonodos fixos \\
\hline \multicolumn{2}{|c|}{ Metástase (M) } \\
\hline MO: & Sem evidências de metástase distante \\
\hline M1: & Metástase distante detectada \\
\hline \multicolumn{2}{|c|}{ Tumor primário $(\mathrm{T})$} \\
\hline Tis: & Tumor pré invasivo (in situ) \\
\hline T0: & Sem evidências de tumor \\
\hline T1: & Tumor $<2$ cm de diâmetro \\
\hline T2: & Tumor entre $2-5 \mathrm{~cm}$ de diâmetro \\
\hline T3: & Tumor $>5 \mathrm{~cm}$ de diâmetro \\
\hline T4: & Tumor invadindo outras estruturas como fáscia, osso ou cartilagem \\
\hline \multicolumn{2}{|c|}{ Linfonodo $(\mathrm{N})$} \\
\hline N0: & Sem evidências de envolvimento de linfonodo regional \\
\hline \multirow[t]{3}{*}{ N1: } & Linfonodo ipsilateral móvel \\
\hline & N1a: sem aumento de volume \\
\hline & N1b: com aumento de volume \\
\hline \multirow[t]{3}{*}{ N2: } & Linfonodo contralateral ou bilateral móvel \\
\hline & N2a: sem aumento de volume \\
\hline & N2b: com aumento de volume \\
\hline N3: & Linfonodos fixos \\
\hline \multicolumn{2}{|c|}{ Metástase (M) } \\
\hline M0: & Sem evidências de metástase distante \\
\hline M1: & Metástase distante detectada \\
\hline
\end{tabular}

Quadro 1- Estadiamento clínico para neoplasias cutâneas em cães segundo sistema TNM ${ }^{23}$

\section{Resultados}

A análise histológica das lesões inclusas no estudo $(\mathrm{n}=34)(100 \%)$ revelou sete melanomas malignos em saco conjuntival, dois orais e um interdigital.
Foram também verificados sete carcinomas espinocelulares, cinco adenocarcinomas hepatoides, três adenomas hepatoides, três adenocarcinomas sebáceos, dois melanocitomas palpebrais, um carcinoma de células basais, um plasmocitoma 


\begin{tabular}{|c|c|c|c|}
\hline \multicolumn{4}{|c|}{ Tumor primário $(\mathrm{T})$} \\
\hline Tis: & \multicolumn{3}{|c|}{ Tumor pré invasivo (in situ) } \\
\hline T1: & \multicolumn{3}{|c|}{ Tumor $<2 \mathrm{~cm}$ de diâmetro } \\
\hline & \multicolumn{3}{|c|}{ T1a: sem evidências de invasão óssea } \\
\hline & \multicolumn{3}{|c|}{ T1b: com evidências de invasão óssea } \\
\hline T2: & \multicolumn{3}{|c|}{ Tumor entre 2 - $4 \mathrm{~cm}$ de diâmetro } \\
\hline & \multicolumn{3}{|c|}{ T2a: sem evidências de invasão óssea } \\
\hline & \multicolumn{3}{|c|}{ T2b: com evidências de invasão óssea } \\
\hline T3: & \multicolumn{3}{|c|}{ Tumor $>4$ cm de diâmetro } \\
\hline \multicolumn{4}{|c|}{ Linfonodo $(\mathrm{N})$} \\
\hline N0: & \multicolumn{3}{|c|}{ Sem metástase para linfonodo regional } \\
\hline N1: & \multicolumn{3}{|c|}{ Linfonodo ipsilateral móvel } \\
\hline & \multicolumn{3}{|c|}{ N1a: sem evidências de metástase para linfonodo } \\
\hline & \multicolumn{3}{|c|}{ N1b: com evidências de metástase para linfonodo } \\
\hline N2: & \multicolumn{3}{|c|}{ Linfonodo contralateral ou bilateral móvel } \\
\hline & \multicolumn{3}{|c|}{ N2a: sem evidências de metástase para linfonodos } \\
\hline & \multicolumn{3}{|c|}{ N2b: com evidências de metástase para linfonodos } \\
\hline N3: & \multicolumn{3}{|c|}{ Linfonodos fixos } \\
\hline \multicolumn{4}{|c|}{$\begin{array}{l}\text { Metástase distante } \\
\text { (M) }\end{array}$} \\
\hline M0: & \multicolumn{3}{|c|}{ Sem evidências de metástase distante } \\
\hline M1: & \multicolumn{3}{|c|}{ Metástase distante detectada } \\
\hline Grupos & Tumor $(\mathrm{T})$ & Linfonodo $(\mathrm{N})$ & Metástase (M) \\
\hline $\mathrm{I}$ & T1 & N0, N1a, N2a & MO \\
\hline II & $\mathrm{T} 2$ & N0, N1a,N1b & MO \\
\hline III & T3 & No, N1a,N1b & MO \\
\hline IV & $\mathrm{T} 1, \mathrm{~T} 2, \mathrm{~T} 3$ & $\mathrm{~N} 1 \mathrm{~b}$ & MO \\
\hline & $\mathrm{T} 1, \mathrm{~T} 2, \mathrm{~T} 3$ & $\mathrm{~N} 2 \mathrm{~b}, \mathrm{~N} 3$ & MO \\
\hline & $\mathrm{T} 1, \mathrm{~T} 2, \mathrm{~T} 3$ & $\mathrm{~N} 1, \mathrm{~N} 2, \mathrm{~N} 3$ & M1 \\
\hline
\end{tabular}

Quadro 2- Estadiamento clínico para neoplasias orais em cães segundo sistema TNM ${ }^{24}$

tegumentar solitário, um epitelioma palpebral e um adenoma sebáceo. Os exames físico, laboratoriais e por imagem não indiciaram a existência de metástases adjacentes e/ou distantes, excetuandose um caso de melanoma interdigital propagado para linfonodo poplíteo ipsilateral, subsequentemente excisado. As informações relativas à caracterização dos animais, volume e localização anatômica, assim como diagnóstico histopatológico e estadiamento clínico de todas as neoformaçóes, encontram-se pormenorizadas na tabela 1 .
Inexistiram complicaçóes e/ou efeitos adversos decorrentes da administração intralesional de sulfato de bleomicina e/ou da eletroporação. Espasmos musculares involuntários e transitórios foram observados durante a aplicaçáo de pulsos elétricos, os quais cessaram após o término do procedimento. Não se evidenciaram lesões térmicas e/ou hemorrágicas imediatas decorrentes do contato dos eletrodos com tecido ou órgáo submetido ao protocolo.

Sete dias após a eletroquimioterapia, observou-se discreta diminuição de volume das neoplasias, as quais em alguns casos exibiam erosões encimadas por crostas hemáticas. Aos 
Tabela 1- Caracterização dos animais e aspectos clínicos e histopatológicos de neoplasias cutâneas e de mucosas. FMV/UNIP - São Paulo - 2009

\begin{tabular}{|c|c|c|c|c|c|c|c|}
\hline Cão & Raça & Sexo & $\begin{array}{l}\text { Idade } \\
\text { (anos) }\end{array}$ & $\begin{array}{c}\text { Volume } \\
\text { tumoral } \\
\text { AxLxP (cm) }\end{array}$ & $\begin{array}{l}\text { Localização } \\
\text { anatômica }\end{array}$ & Histopatológico & $\begin{array}{c}\text { Estadiamento } \\
\text { clínico }\end{array}$ \\
\hline 1 & Pastor Alemão & $M$ & 8 & $1.0 \times 1.0 \times 0.5$ & Saco conjuntival & Melanoma melânico & T1 N0 M0 \\
\hline 2 & Pastor Alemão & M & 9 & $1.2 \times 0.6 \times 0.4$ & Saco conjuntival & Melanoma melânico & T1 No M0 \\
\hline 3 & Weimaraner & $\mathrm{F}$ & 11 & $2.2 \times 1.0 \times 1.0$ & Saco conjuntival & Melanoma amelânico & T2 NO MO \\
\hline 4 & Weimaraner & $\mathrm{F}$ & 10 & $1.8 \times 1.3 \times 1.0$ & Saco conjuntival & Melanoma amelânico & T1 No Mo \\
\hline 5 & Weimaraner & M & 8 & $4.0 \times 3.7 \times 2.7$ & Saco conjuntival & Melanoma amelânico & T2 NO MO \\
\hline 6 & Teckel & $\mathrm{F}$ & 10 & $1.0 \times 0.7 \times 0.6$ & Saco conjuntival & Melanoma amelânico & T1 No Mo \\
\hline 7 & Labrador & $\mathrm{F}$ & 7 & $0.5 \times 0.3 \times 0.2$ & Saco conjuntival & Melanoma amelânico & T1 No Mo \\
\hline 8 & Mestiço & $\mathrm{F}$ & 12 & $1.4 \times 0.6 \times 0.8$ & Mucosa oral & Melanoma amelânico & T1 No M0 (Gl) \\
\hline 9 & Poodle & M & 10 & $1.6 \times 0.8 \times 0.4$ & Mucosa oral & Melanoma melânico & T1 No Mo (GI) \\
\hline 10 & Schnauzer & M & 8 & $1.4 \times 0.9 \times 0.7$ & Interdigital & Melanoma melânico & T1 N1b M0 \\
\hline 11 & $\begin{array}{l}\text { Husky } \\
\text { Siberiano }\end{array}$ & M & 11 & $0.9 \times 0.9 \times 0.3$ & Perianal & $\begin{array}{l}\text { Carcinoma espino- } \\
\text { celular }\end{array}$ & T1 No Mo \\
\hline 12 & Mestiço & $\mathrm{F}$ & 10 & $1.5 \times 1.5 \times 0.9$ & Palpebral & $\begin{array}{c}\text { Carcinoma espino- } \\
\text { celular }\end{array}$ & T1 No M0 \\
\hline 13 & Mestiço & $\mathrm{F}$ & 12 & $0.5 \times 0.3 \times 0.3$ & Palpebral & $\begin{array}{l}\text { Carcinoma espino- } \\
\text { celular }\end{array}$ & T1 No M0 \\
\hline 14 & Cocker Spaniel & M & 5 & $1.5 \times 1.5 \times 1.5$ & Lombar & $\begin{array}{c}\text { Carcinoma espino- } \\
\text { celular }\end{array}$ & T1 No M0 \\
\hline 15 & Poodle & $\mathrm{F}$ & 16 & $2.0 \times 1.6 \times 2.8$ & Cauda & $\begin{array}{c}\text { Carcinoma espino- } \\
\text { celular }\end{array}$ & T2 NO MO \\
\hline 16 & Pit Bull & M & 12 & $1.5 \times 2.0 \times 1.5$ & Lombar & $\begin{array}{l}\text { Carcinoma espino- } \\
\text { celular }\end{array}$ & T1 No M0 \\
\hline 17 & Poodle & $\mathrm{F}$ & 7 & $2.0 \times 1.3 \times 2.0$ & Facial & $\begin{array}{l}\text { Carcinoma espino- } \\
\text { celular }\end{array}$ & T2 NO MO \\
\hline 18 & Pastor Alemão & M & 12 & $1.5 \times 1.5 \times 1.5$ & Perianal & $\begin{array}{c}\text { Adenocarcinoma } \\
\text { hepatoide }\end{array}$ & T1 No Mo \\
\hline 19 & Cocker Spaniel & M & 10 & $0.5 \times 0.5 \times 0.3$ & Perianal & $\begin{array}{l}\text { Adenocarcinoma } \\
\text { hepatoide }\end{array}$ & T1 No Mo \\
\hline 20 & Mestiço & M & 10 & $1.3 \times 1.3 \times 1.0$ & Perianal & $\begin{array}{l}\text { Adenocarcinoma } \\
\text { hepatoide }\end{array}$ & T1 No M0 \\
\hline 21 & Cocker Spaniel & M & 12 & $1.5 \times 1.7 \times 0.7$ & Perianal & $\begin{array}{l}\text { Adenocarcinoma } \\
\text { hepatoide }\end{array}$ & T1 No Mo \\
\hline 22 & Poodle & M & 13 & $2.2 \times 2.3 \times 2.0$ & Perianal & $\begin{array}{l}\text { Adenocarcinoma } \\
\text { hepatoide }\end{array}$ & T2 NO MO \\
\hline 23 & $\begin{array}{l}\text { Husky } \\
\text { Siberiano }\end{array}$ & M & 11 & $3.0 \times 1.5 \times 1.0$ & Perianal & Adenoma hepatoide & T2 NO MO \\
\hline 24 & $\begin{array}{l}\text { Husky } \\
\text { Siberiano }\end{array}$ & M & 14 & $4.3 \times 2.9 \times 3.7$ & Perianal & Adenoma hepatoide & T2 NO MO \\
\hline 25 & Cocker Spaniel & M & 10 & $1.0 \times 0.9 \times 0.3$ & Perianal & Adenoma hepatoide & T1 No M0 \\
\hline 26 & Akita & M & 12 & $1.1 \times 1.0 \times 0.5$ & Torácica & $\begin{array}{c}\text { Adenocarcinoma } \\
\text { sebáceo }\end{array}$ & T1 No M0 \\
\hline 27 & Cocker Spaniel & M & 11 & $0.8 \times 0.6 \times 0.3$ & Facial & $\begin{array}{l}\text { Adenocarcinoma } \\
\text { sebáceo }\end{array}$ & T1 No Mo \\
\hline 28 & Cocker Spaniel & M & 12 & $2.9 \times 2.1 \times 0.2$ & $\begin{array}{l}\text { Pavilhão } \\
\text { auricular }\end{array}$ & $\begin{array}{l}\text { Adenocarcinoma } \\
\text { sebáceo }\end{array}$ & T2 NO MO \\
\hline 29 & Akita & M & 12 & $0.5 \times 0.5 \times 0.5$ & Palpebral & Melanocitoma & T1 No Mo \\
\hline 30 & Weimaraner & M & 8 & $0.8 \times 0.8 \times 0.6$ & Palpebral & Melanocitoma & T1 No Mo \\
\hline 31 & Poodle & M & 2 & $1.0 \times 0.8 \times 0.7$ & Cervical & $\begin{array}{c}\text { Carcinoma de células } \\
\text { basais }\end{array}$ & T1 No M0 \\
\hline 32 & Pit Bull & $\mathrm{F}$ & 7 & $0.6 \times 0.6 \times 0.4$ & Perianal & $\begin{array}{l}\text { Plasmocitoma } \\
\text { tegumentar }\end{array}$ & T1 No MO \\
\hline 33 & Poodle & M & 15 & $0.7 \times 0.4 \times 0.6$ & Palpebral & Epitelioma & T1 No Mo \\
\hline 34 & Poodle & $\mathrm{F}$ & 5 & $1.4 \times 1.4 \times 0.7$ & Facial & Adenoma sebáceo & T1 No Mo \\
\hline
\end{tabular}


14 dias, evidenciou-se remissão parcial das neoformaçóes e persistência de áreas erodidas ainda encimadas pelo mesmo tipo de crostas. Aos 21 dias de observaçáo, houve acentuada regressão neoplásica por vezes acompanhada por discreta solução de descontinuidade tegumentar. A reavaliação macroscópica 30 dias após a primeira sessão da terapia, apontou remissão tumoral integral em nove pacientes (Figura 1). Neoplasias as quais exibiram regressão incompleta após uma sessão ( $\mathrm{n}$ = 21) foram subsequentemente submetidas a novas aplicaçóes do protocolo, em intervalos mensais, até a remissão total (Figuras 2 e 3).

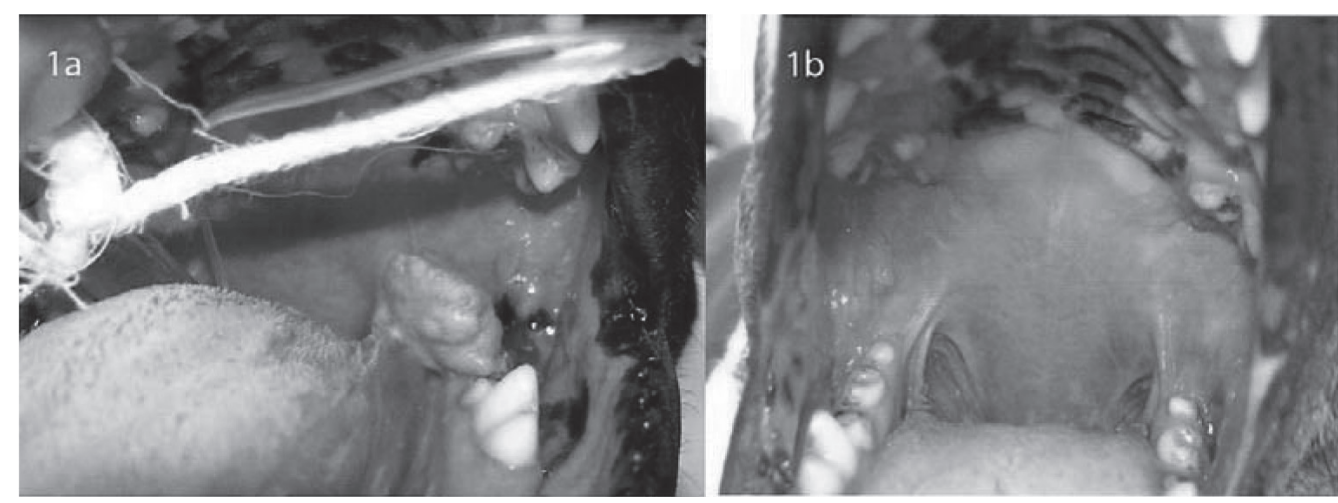

Figura 1- a) Aspecto macroscópico de melanoma amelânico em mucosa oral (animal no 8, pré terapia); b) Remissão neoplásica integral 30 dias após a primeira sessão eletroquimioterápica

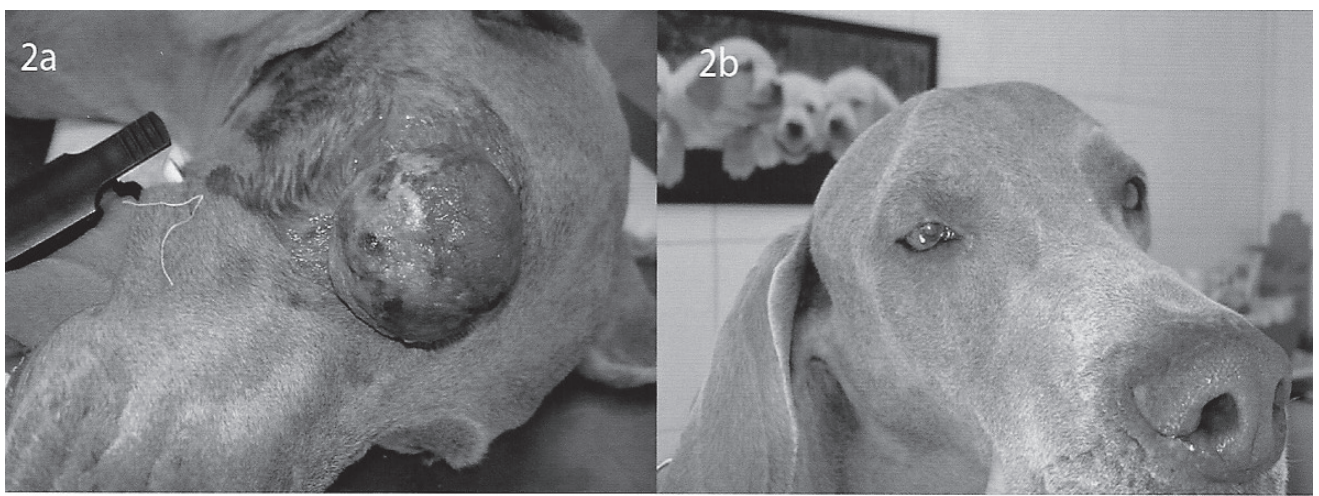

Figura 2- a) Aspecto macroscópico de melanoma amelânico em saco conjuntival (animal no 5, pré terapia); b) Remissão neoplásica integral após três sessões eletroquimioterápicas (90 dias)

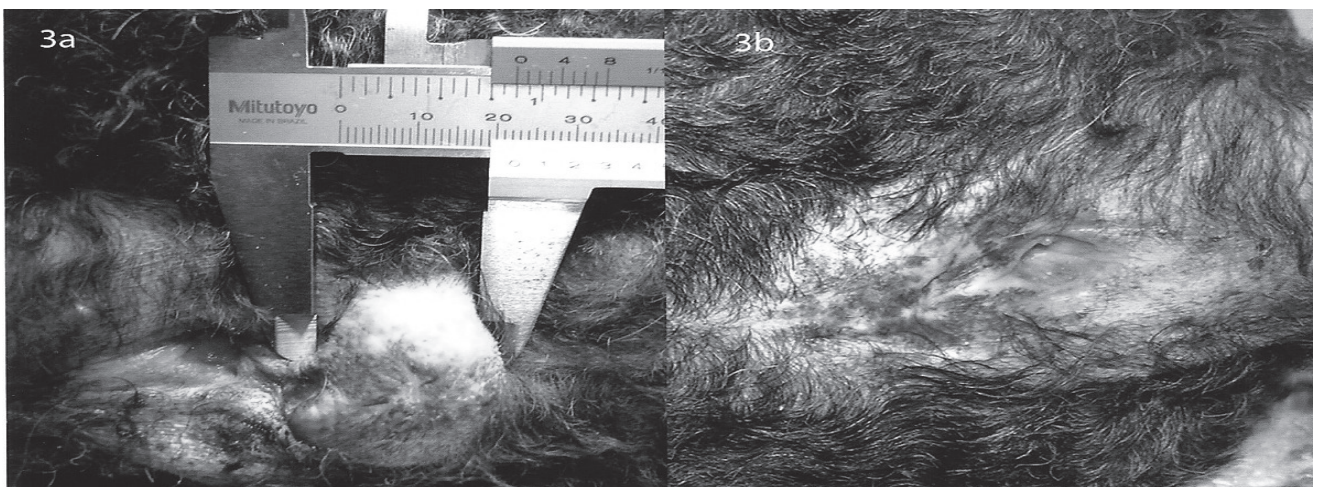

Figura 3- a) Aspecto macroscópico de adenocarcinoma hepatoide (animal n 22, pré terapia); b) Remissão neoplásica integral após três sessões eletroquimioterápicas (90 dias) 
O número de sessôes eletroquimioterápicas necessárias à remissão neoplásica integral oscilou entre os animais (uma a três) (Tabela 2). A maioria dos cáes apresentou regressão tumoral completa $(\mathrm{n}=$ $30)(88,3 \%)$. Apenas quatro neoformaçóes
$(11,7 \%)$ não apresentaram resposta a contento após duas sessôes, respectivamente representadas por três carcinomas espinocelulares e um adenocarcinoma sebáceo, posteriormente excisados (Tabela 2 e Figura 4).

Tabela 2- Dados referentes à quantidade de sessões eletroquimioterápicas e resposta tumoral. FMV/UNIP - São Paulo - 2009

(Continua)

\begin{tabular}{|c|c|c|c|c|c|}
\hline Cão n- & $\begin{array}{c}\text { Volume } \\
\text { tumoral } \\
\text { AxLxP (cm) }\end{array}$ & $\begin{array}{l}\text { Localização } \\
\text { anatômica }\end{array}$ & Histopatológico & $\mathrm{n}-$ de sessões & Resposta tumoral \\
\hline 1 & $1.0 \times 1.0 \times 0.5$ & Saco conjuntival & $\begin{array}{l}\text { Melanoma } \\
\text { melânico }\end{array}$ & 2 & RT \\
\hline 2 & $1.2 \times 0.6 \times 0.4$ & Saco conjuntival & $\begin{array}{l}\text { Melanoma } \\
\text { melânico }\end{array}$ & 2 & RT \\
\hline 3 & $2.2 \times 1.0 \times 1.0$ & Saco conjuntival & $\begin{array}{l}\text { Melanoma } \\
\text { amelânico }\end{array}$ & 3 & RT \\
\hline 4 & $1.8 \times 1.3 \times 1.0$ & Saco conjuntival & $\begin{array}{l}\text { Melanoma } \\
\text { amelânico }\end{array}$ & 3 & RT \\
\hline 5 & $4.0 \times 3.7 \times 2.7$ & Saco conjuntival & $\begin{array}{l}\text { Melanoma } \\
\text { amelânico }\end{array}$ & 3 & RT \\
\hline 6 & $1.0 \times 0.7 \times 0.6$ & Saco conjuntival & $\begin{array}{l}\text { Melanoma } \\
\text { amelânico }\end{array}$ & 2 & RT \\
\hline 7 & $0.5 \times 0.3 \times 0.2$ & Saco conjuntival & $\begin{array}{l}\text { Melanoma } \\
\text { amelânico }\end{array}$ & 1 & RT \\
\hline 8 & $1.4 \times 0.6 \times 0.8$ & Mucosa oral & $\begin{array}{l}\text { Melanoma } \\
\text { amelânico }\end{array}$ & 1 & RT \\
\hline 9 & $1.6 \times 0.8 \times 0.4$ & Mucosa oral & $\begin{array}{l}\text { Melanoma } \\
\text { melânico }\end{array}$ & 3 & RT \\
\hline 10 & $1.4 \times 0.9 \times 0.7$ & Interdigital & $\begin{array}{l}\text { Melanoma } \\
\text { melânico }\end{array}$ & 3 & RT \\
\hline 11 & $0.9 \times 0.9 \times 0.3$ & Perianal & $\begin{array}{l}\text { Carcinoma espino- } \\
\text { celular }\end{array}$ & 1 & RT \\
\hline 12 & $1.5 \times 1.5 \times 0.9$ & Palpebral & $\begin{array}{l}\text { Carcinoma espino- } \\
\text { celular }\end{array}$ & 2 & NR \\
\hline 13 & $0.5 \times 0.3 \times 0.3$ & Palpebral & $\begin{array}{l}\text { Carcinoma espino- } \\
\text { celular }\end{array}$ & 1 & RT \\
\hline 14 & $1.5 \times 1.5 \times 1.5$ & Lombar & $\begin{array}{l}\text { Carcinoma espino- } \\
\text { celular }\end{array}$ & 2 & RT \\
\hline 15 & $2.0 \times 1.6 \times 2.8$ & Cauda & $\begin{array}{l}\text { Carcinoma espino- } \\
\text { celular }\end{array}$ & 2 & NR \\
\hline 16 & $1.5 \times 2.0 \times 1.5$ & Lombar & $\begin{array}{l}\text { Carcinoma espino- } \\
\text { celular }\end{array}$ & 2 & RT \\
\hline 17 & $2.0 \times 1.3 \times 2.0$ & Facial & $\begin{array}{l}\text { Carcinoma espino- } \\
\text { celular }\end{array}$ & 2 & NR \\
\hline 18 & $1.5 \times 1.5 \times 1.5$ & Perianal & $\begin{array}{c}\text { Adenocarcinoma } \\
\text { hepatoide }\end{array}$ & 2 & RT \\
\hline
\end{tabular}




\begin{tabular}{|c|c|c|c|c|c|}
\hline 19 & $0.5 \times 0.5 \times 0.3$ & Perianal & $\begin{array}{l}\text { Adenocarcinoma } \\
\text { hepatoide }\end{array}$ & 2 & RT \\
\hline 20 & $1.3 \times 1.3 \times 1.0$ & Perianal & $\begin{array}{l}\text { Adenocarcinoma } \\
\text { hepatoide }\end{array}$ & 2 & RT \\
\hline 21 & $1.5 \times 1.7 \times 0.7$ & Perianal & $\begin{array}{l}\text { Adenocarcinoma } \\
\text { hepatoide }\end{array}$ & 2 & RT \\
\hline 22 & $2.2 \times 2.3 \times 2.0$ & Perianal & $\begin{array}{l}\text { Adenocarcinoma } \\
\text { hepatoide }\end{array}$ & 3 & RT \\
\hline 23 & $3.0 \times 1.5 \times 1.0$ & Perianal & Adenoma hepatoide & 2 & RT \\
\hline 24 & $4.3 \times 2.9 \times 3.7$ & Perianal & Adenoma hepatoide & 3 & RT \\
\hline 25 & $1.0 \times 0.9 \times 0.3$ & Perianal & Adenoma hepatoide & 1 & RT \\
\hline 26 & $1.1 \times 1.0 \times 0.5$ & Torácica & $\begin{array}{c}\text { Adenocarcinoma } \\
\text { sebáceo }\end{array}$ & 3 & RT \\
\hline 27 & $0.8 \times 0.6 \times 0.3$ & Facial & $\begin{array}{l}\text { Adenocarcinoma } \\
\text { sebáceo }\end{array}$ & 3 & RT \\
\hline 28 & $2.9 \times 2.1 \times 0.2$ & $\begin{array}{l}\text { Pavilhão auri- } \\
\text { cular }\end{array}$ & $\begin{array}{c}\text { Adenocarcinoma } \\
\text { sebáceo }\end{array}$ & 2 & NR \\
\hline 29 & $0.5 \times 0.5 \times 0.5$ & Palpebral & Melanocitoma & 1 & RT \\
\hline 30 & $0.8 \times 0.8 \times 0.6$ & Palpebral & Melanocitoma & 1 & RT \\
\hline 31 & $1.0 \times 0.8 \times 0.7$ & Cervical & $\begin{array}{l}\text { Carcinoma de } \\
\text { células basais }\end{array}$ & 2 & RT \\
\hline 32 & $0.6 \times 0.6 \times 0.4$ & Perianal & $\begin{array}{l}\text { Plasmocitoma } \\
\text { tegumentar }\end{array}$ & 1 & RT \\
\hline 33 & $0.7 \times 0.4 \times 0.6$ & Palpebral & Epitelioma & 1 & RT \\
\hline 34 & $1.4 \times 1.4 \times 0.7$ & Facial & Adenoma sebáceo & 2 & RT \\
\hline
\end{tabular}

RT: Remissão total, NR: Não responsivo

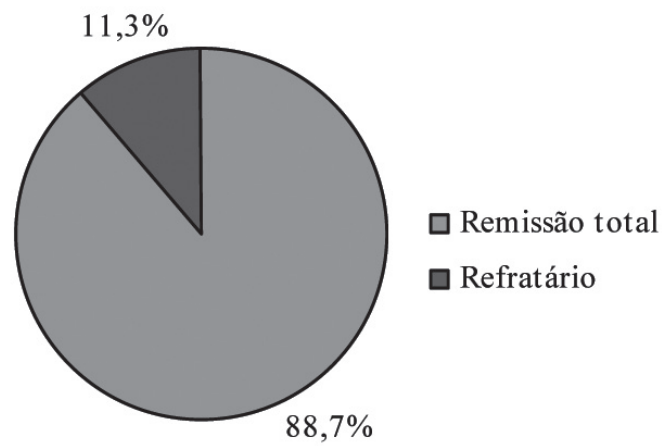

Figura 4 - Percentual de resposta tumoral após protocolo eletroquimioterápico 
Todos os pacientes foram reavaliados em retornos bimestrais por meio de exames físico e complementares por período de doze meses, não exibindo indícios orgânicos, laboratoriais e/ou por imagem de recidiva neoplásica e/ou metástase(s) adjacente(s) el ou distante(s) neste intervalo de tempo.

\section{Discussão}

A despeito de todo conhecimento genético e molecular dos tumores até então obtido, dados estatísticos atuais demonstram que os mesmos figuram entre as principais causas de óbito em cães ${ }^{1}$. Assim sendo, há crescente demanda pela realização de pesquisas adicionais, objetivando a busca por novas opçóes terapêuticas em medicina oncológica $^{1,2,8,10}$. A eletroquimioterapia, procedimento que alia o uso de fármacos antineoplásicos à aplicação regional de pulsos elétricos, facilita a entrada destes agentes ao meio intracelular pelo princípio da eletropermeabilização otimizando sua atividade citotóxica em baixas doses, minimizando assim, a ocorrência de efeitos adversos ${ }^{3,4,9,12,14,15,16,20}$. A bibliografia reunida caracterizou-a como modalidade aplicável, eficaz e segura na terapêutica antitumoral em cães ${ }^{16,17,18,19,20,21}$.

Neste estudo, avaliou-se um protocolo eletroquimioterápico no qual se utilizou sulfato de bleomicina, pela via intralesional, em diversos tipos tumorais de origem epitelial e mesenquimal, situados em pele ou mucosas, a exceção dos sarcomas. A literatura disponibiliza informaçôes referentes ao uso de inúmeros agentes antineoplásicos em associação à eletroporação. Todavia, existe um consenso entre os autores relativamente à potencializaçáo da citotoxicidade exercida por cisplatina e bleomicina, uma vez combinadas à administração de pulsos elétricos ${ }^{4,12,14}$. A eletroquimioterapia é indicada no tratamento de diversos tipos de neoplasias cutâneas e de membranas mucosas, independentemente de sua histologia ${ }^{4,12,14,19,20,21}$. Entretanto, pesquisas prévias apontaram que naqueles tumores ricos em fibras colágenas, a exemplo dos sarcomas, existe dificuldade na propagação dos pulsos elétricos assim como do fármaco antineoplásico, com consequente refratariedade ao protocolo ${ }^{25,26,27}$.

A aplicaçáo intralesional do quimioterápico sulfato de bleomicina demonstrouse efetiva e isenta de efeitos colaterais decorrentes, dado igualmente verificado e descrito em estudos preliminares $3,4,9,14,17,20$. Todavia, cabe destacar que em quatro neoplasias erodo-ulceradas, houve dificuldade na difusão intralesional do fármaco, sobretudo sugerida por sua refratariedade ao protocolo. Trabalhos prévios apontaram que a administração endovenosa de sulfato de bleomicina representa a via de eleição nos casos de neoformaçóes volumosas e/ou erodo-ulceradas, pois a tais características macroscópicas foi atribuida a limitaçáo da inadequada concentraçáo do agente antineoplásico, quando localmente aplicado ${ }^{4,9,20}$. Entretanto, nesta pesquisa, respeitando-se a metodologia preconizada, optou-se pela excisáo cirúrgica de neoplasias refratárias ao procedimento eletroquimioterápico.

Lesôes teciduais térmicas advindas da eletroporação inexistiram nos animais da amostragem considerada. Tal fenômeno, por outros autores detectado em inexpressivo número de casos, foi naquelas situações, diretamente atribuido à amplitude, duração, frequência e quantidade administrada de pulsos elétricos ${ }^{9,18,20}$.

Nas neoformaçóes submetidas à eletroquimioterapia nesta pesquisa, não houve eventos hemorrágicos macroscópicos imediatos. Ressalte-se que à eletroporaçáo, foi referida a propriedade de desestruturação endotelial vascular neoplásica com consequente reduçáo em seu aporte sanguineo, assim propiciando redução do risco de hemorragia pela adentrada dos eletrodos no tumor ${ }^{5,12,13,21,22}$.

Durante a administração de pulsos elétricos, foram observados espasmos musculares regionais involuntários, instantaneamente cessados após o término do procedimento. Tal fato propicia desconforto de ínfima magnitude ao paciente ${ }^{4,6,7,11,20,21}$. 
O grupo e dosagem dos fármacos antitumorais empregados, características físico-quimicas dos eletrodos, assim como a intensidade, número e tipo de onda por estes produzida, diferem entre os trabalhos compulsados, resultando complexo o cotejamento referente aos aspectos supracitados $3,4,6,7,8,14,16,17,18,19$. Contudo, sulfato de bleomicina, na dose de $1 \mathrm{U} /$ $\mathrm{cm}^{3}$ de tumor, associado à administração regional de pulsos elétricos com tensão de $1000 \mathrm{~V}$, por 100 microsegundos, em onda quadrada unipolar, totalizando oito pulsos, demonstrou-se eficaz na remissão das neoplasias aqui consideradas.

O número de sessóes eletroquimioterápicas para a regressão tumoral integral oscilou entre os pacientes (uma a três). Segundo a bibliografia consultada, a repetição do protocolo encontra-se diretamente influenciada pelo tipo e volume $\mathrm{da}$ neoformação $0^{4,9,14,17}$.

O monitoramento clínico, conduzido pelo período de doze meses na totalidade de cães deste estudo, revelou não haver indícios de recidiva e/ou propagação neoplásica. O potencial metastático tumoral está condicionado a uma miríade de fatores, dentre os quais se enfocam tipo, volume e localização anatômica da neoformação, protocolo(s) terapêutico(s) utilizado(s) e salubridade imunológica ${ }^{1}$. Sabe-se que a eletroquimioterapia, ainda que por mecanismos não completamente elucidados, promove estimulação imunitária celular e humoral, minimizando então, a ocorrência de recidivas e/ou metástases ${ }^{4,10,14,16,19,21}$.

\section{Conclusões}

O confronto dos resultados aqui obtidos às informaçóes acessadas na bibliografia consultada permitiu concluir que a eletroquimioterapia representa uma modalidade terapêutica aplicável, eficaz e segura para emprego em medicina oncológica veterinária.

Dentre as vantagens intrínsecas ao procedimento estudado, enfocam-se rapidez e praticidade em sua execução, ausência de toxicidade atribuível à administração intralesional do fármaco sulfato de bleomicina, baixa onerosidade e inexistência de complicaçôes trans e pós-terapêuticas.

\section{Employment of electrochemotherapy in canine neoplasms of epithelial or mesenchymal origin located in the skin or mucosal membranes}

\begin{abstract}
Electrochemotherapy is characterized as a protocol which combines the use of antineoplastic agents with localized application of electric pulses to improve the intracellular concentration of these agents, increasing, thus, its cytotoxic action. Bleomycin, an antibiotic agent with antineoplastic properties, is a hydrophilic molecule, having a restricted transport through the cellular membrane. However, when it is administered intralesionally or intravenously and associated to electroporation, its cytotoxicity is maximized. In this study 34 dogs were utilized and they were affected by a single lesion of epithelial or mesenchymal origin, located in the skin or mucosal membranes. The electrochemotherapy protocol was standardized using intralesional bleomycin sulfate at a dose of $1 \mathrm{U} / \mathrm{cm}^{3}$ of tumoral area. Electroporation was performed using an electrode composed of needles and electric pulses with a $1000 \mathrm{~V}$ voltage, in unipolar square wave and 100 microseconds duration, totalizing eight cycles. There was complete neoplastic remission in 30 dogs (88.3\%) and refractoriness to the protocol in four animals $(11.7 \%)$. There were
\end{abstract}

Keywords:

Dog.

Neoplasia.

Electroporation.

Electrochemotherapy. 
no complications or side effects associated with the procedure. The protocol studied in this work showed to be feasible, effective and safe for antineoplastic therapy in dogs.

\section{Referências}

1 REIF, J. S. The epidemiology and incidence of cancer. In: WITHROW, S. J.; VAIL, D. M. Withrow \& MacEwen's small animal clinical oncology. 4. ed. St. Louis: Saunders Elsevier, 2007. p. 68-76.

2 OLIVEIRA, L. O.; TELLÓ, M. Pesquisas, em nível mundial, referentes à utilização da corrente contínua no tratamento do câncer. In: TELLÓ, M.; RAIZER, A.; BUZAID, A. C.; DOMENGE, C.; DIAS, G. A. D.; ALMAGUER, H. D.; OLIVEIRA, L. O.; FARBER, P. L.; OLIVEIRA, R. T.; SILVA, V. D. O uso da corrente elétrica no tratamento do câncer. 3. ed. Porto Alegre: EDIPUCRS, 2004. p. 77-96.

3 LARKIN, J. O.; COLLINS, C. G.; AARONS, S.; TANGNEY, M.; WHELAN, M.; O'REILY, S.; BREATHNACH, O.; SODEN, D. M.; O'SULLIVAN, G. C. Electrochemotherapy: aspects of preclinical development and early clinical experience. Annals of Surgery, v. 245, n. 3, p. 469-479, 2007.

4 GOTHELF, A.; MIR, L. M.; GEHL, J. Electrochemotherapy: results of cancer treatment using enhanced delivery of bleomycin by electroporation. Cancer Treatment Reviews, v. 29, n. 5, p. 371-387, 2003.

5 DUJARDIN, N.; STAES, E.; KALIA, Y.; CLARYS, P.; GUY, R.; PRÉAT, V. In vivo assessment of skin electroporation using square wave pulses. Journal of Controlled Release, v. 79, n. 1-3, p. 219-227, 2002.

6 LEBAR, A. M.; SERŠA, G.; KRANJC, S.; GROŠELJ, A.; MIKLAVČIČ, D. Optimization of pulse parameters in vitro for in vivo electrochemotherapy. Anticancer Research, v. 22, n. 3, p. 1731-1736, 2002.

7 PUCIHAR, G.; MIR, L. M.; MIKLAVČIČ, D. The effect of pulse repetition frequency on the uptake into electropermeabilized cells in vitro with possible applications in electrochemotherapy. Bioelectrochemistry, v. 57, n. 2, p. 167-172, 2002.

8 CHEN, C.; SMYE, S. W.; ROBINSON, M. P.; EVANS, J. A. Membrane electroporation theories: a review. Medical and Biological Engineering and Computing, v. 44, n. 1-2, p. 5-14, 2006.

9 MIR, L. M.; GLASS, L. F.; SERŠA, G.; TEISSIÉ, J.; DOMENGE, C.; MIKLAVČIČ, D.; JAROSZESKI, M. J.; ORLOWSKI, S.; REINTGEN, D. S.; RUDOLF, Z.; BELEHRADEK, M.; GILBERT, R. A.; ROLS, M. P.; BELEHRADEK JR., J.; BACHAUD, J. M.; De CONTI, R. C.; ŠTABUC, B.; ČEMAŽAR, M.; CONINX, P.; HELLER, $R$. Effective treatment of cutaneous and subcutaneous malignant tumours by electrochemotherapy. British Journal of Cancer, v. 77, n. 12, p. 2336-2342, 1998.
10 CIRIAL, H. C.; LÓPEZ, D. S. La electroquimioterapia: una nueva alternativa terapéutica en la oncología. Revista Cubana de Oncología, v. 17, n. 3, p. 188-194, 2001.

11 MIKLAVČIČ, D.; PUCIHAR, G.; PAVLOVEC, M.; RIBARIČ, S.; MALI, M.; LEBAR, A. M.; PETKOVŠEK, M.; NASTRAN, J.; KRANJC, S.; ČEMAŽAR, M.; SERŠA, G. The effect of high frequency electric pulses on muscle contractions and antitumor efficiency in vivo for a potential use in clinical electrochemotherapy. Bioelectrochemistry, v. 65, n. 2, p. 121-128, 2005.

12 GIARDINO, R.; FINI, M.; BONAZZI, V.; CADOSSI, R.; NICOLINI, A.; CARPI, A. Electrochemotherapy: a novel approach to the treatment of metastatic nodules on the skin and subcutaneous tissues. Biomedicine and Pharmacotherapy, v. 60, n. 8, p. 458-462, 2006.

13 SERŠA, G.; ČEMAŽAR, M.; PARKINS, C. S.; CHAPLIN, D. J. Tumour blood flow changes induced by application of electric pulses. European Journal of Cancer, v. 35, n. 4, p. 672-677, 1999.

14 SERŠA, G.; ČEMAŽAR, M.; MIKLAVČIČ, D.; RUDOLF, Z. Electrochemotherapy of tumours. Radiology and Oncology, v. 40, n. 3, p. 163-174, 2006.

15 ČEMAŽAR, M.; MIKLAVČIČ, D.; MIR, L. M.; BELEHRADEK JR., J.; BONNAY, M.; FOURCAULT, D.; SERŠA G. Electrochemotherapy of tumours resistant to cisplatin: a study in a murine tumour model. European Journal of Cancer, v. 37, n. 9, p. 1166-1172, 2001.

16 PARISE JR., O.; TELLÓ, M.; OLIVEIRA, L. O.; ZANELLA, R.; OLIVEIRA, R. T.; SILVA, C. C. F.; LEMES, A.; GIOSO, M. A.; BARBUTO, J. A.; BUZAID, A. C. Efeito antitumoral do campo elétrico potencializando quimioterápicos: estudo no modelo animal. Revista Brasileira de Cirurgia de Cabeça e Pescoço, v. 37, n. 2, p. 76-81, 2008

17 AMINKOV, B.; MANOV, V. Electrochemotherapy: a novel method of treatment of malignant tumours in the dog. Bulgarian Journal of Veterinary Medicine, $v$. 7, n. 4, p. 209-213, 2004.

18 TOZON, N.; KODRE, V.; SERŠA, G.; ČEMAŽAR, $M$. Effective treatment of perianal tumors in dogs with electrochemotherapy. Anticancer Research, v. 25, n. 2A, p. 839-846, 2005.

19 SPUGNINI, E. P.; DRAGONETTI, E.; VINCENZI, B.; ONORI, N.; CITRO, G.; BALDI, A. Pulse-mediated chemotherapy enhances local control and survival in a spontaneous canine model of primary mucosal melanoma. Melanoma Research, v. 16, n. 1, p. 23-27, 2006.

20 HELLER, R.; JAROSZESKI, M. J.; REINTGEN, D. S.; PULEO, C. A.; De CONTI, R. C.; GILBERT, R. A.; 
GLASS, L. F. Treatment of cutaneous and subcutaneous tumors with electrochemotherapy using intralesional bleomycin. Cancer, v. 83, n. 1, p. 148-157, 1998.

21 ČEMAŽAR, M.; TAMZALI, Y.; SERŠA, G.; TOZON, N.; MIR, L. M.; MIKLAVČIČ, D.; LOWE, R.; TEISSIÉ, J. Electrochemotherapy in veterinary oncology. Journal of Veterinary Internal Medicine, v. 22, n. 4, p. 826831, 2008.

22 SERŠA, G.; JARM, T.; KOTNIK, T.; COER, A.; PODKRAJSEK, M.; SENTJURC, M.; MIKLAVČIČ, D.; KADIVEC, M.; KRANJC, S.; SECEROV, A.; ČEMAŽAR, M. Vascular disrupting action of electroporation and electrochemotherapy with bleomycin in murine sarcoma. British Journal of Cancer, v. 98, n. 2, p. 388-398, 2008

23 VAIL, D. M.; WITHROW, S. J. Tumors of the skin and subcutaneous tissues. In: WITHROW, S. J.; VAIL, D. M. Withrow \& MacEwen's small animal clinical oncology. 4. ed. St. Louis: Saunders Elsevier, 2007. p. 375-401.

24 LIPTAK, J. M.; WITHROW, S. J. Cancer of the gastrointestinal tract. In: WITHROW, S. J.; VAIL,
D. M. Withrow \& MacEwen's small animal clinical oncology. 4. ed. St. Louis: Saunders Elsevier, 2007. p. 455-510.

25 SPUGNINI, E. P.; CITRO, G.; D'AVINO, A.; BALDI, A. Potential role of electrochemotherapy for the treatment of soft tissue sarcoma: first insights from preclinical studies in animals. The International Journal of Biochemistry \& Cell Biology, v. 40, n. 2, p. 159-163, 2008.

26 SPUGNINI, E. P.; VINCENZI, B.; BETTI, G.; CORDAHI, F.; DOTSINSKY, I.; MUDROV, N.; CITRO, G.; BALDI, A. Surgery and electrochemotherapy of a high grade soft tissue sarcoma in a dog. Veterinary Record v. 162, n. 6, p. 186-188, 2008.

27 SPUGNINI, E. P.; BALDI, A.; VINCENZI, B.; BONGIORNI, F.; BELLELLI, C.; CITRO, G.; PORRELLO, A. Intraoperative versus postoperative electrochemotherapy in high grade soft tissue sarcomas: a preliminary study in a spontaneous feline model. Cancer Chemotherapy Pharmacology, v. 59, n. 3, p. 375-381, 2007. 